Sportwiss 2013 · 43:90-101

DOI 10.1007/s12662-013-0291-y

Eingegangen: 24. Oktober 2012

Angenommen: 7. März 2013

Online publiziert: 7. Mai 2013

๑) Springer-Verlag Berlin Heidelberg 2013

Torsten Schlesinger • Siegfried Nagel

Institut für Sportwissenschaft, Universität Bern, Bern, Schweiz

\title{
Individuelle und strukturelle Faktoren der Mitgliederbindung im Sportverein
}

Da Sportvereine auf dem Markt für sportbezogene Dienstleistungen ihre Monopolstellung eingebüßt haben und zunehmend in Konkurrenz zu anderen Sportanbietern stehen, stellt die langfristige Bindung der Mitglieder vielfach keine Selbstverständlichkeit mehr dar. Folgt man den aktuellen Sportentwicklungsberichten in Deutschland und der Schweiz (Breuer, 2011; Lamprecht et al. 2012), dann sehen sich zunehmend mehr Sportvereine mit Mitgliederfluktuationen sowie stagnierenden bzw. zurückgehenden Mitgliederzahlen konfrontiert. Die Relevanz stabiler und dauerhafter Mitgliedschaftsbeziehungen ist nicht nur aus ressourcenspezifischer Perspektive (z. B. für die Rekrutierung von freiwilliger Mitarbeit zur Erstellung der Klubgüter) hervorzuheben (Wicker \& Breuer, 2011). Auch vor dem Hintergrund gesellschaftspolitischer Debatten um die Produktion und Aufrechterhaltung von sozialem Kapital in der modernen Bürgergesellschaft wird die zentrale Bedeutung dauerhafter Vereinsmitgliedschaften vielfach herausgestellt (z. B. Braun, 2001; Bühlmann \& Freitag, 2004).

Als Ursachen für die abnehmende Bindungsbereitschaft von Vereinsmitgliedern werden innerhalb der Diskussion um die Entwicklung von Sportvereinen unterschiedliche Erklärungsansätze bemüht:

1. Die zunehmende Instabilität der Mitgliedschaftsbeziehungen in Sportvereinen wird im Zusammenhang mit der Erosion tradierter gemeinschaftlicher Solidarformen sowie der gesellschaftlichen Bindungslosigkeit diskutiert, was v. a. über Prozesse des tiefgreifenden Wertewandels und einer Zunahme individualistischer Selbstentfaltungsansprüche begründet wird (grundlegend Lenk, 1972; Bette, 1993; Rittner, 1986). In diesem Zusammenhang lösen sich traditionelle und wertrationale Mitgliedschaftsbeziehungen zugunsten zweckrationaler Beziehungen auf, wobei Austauschprozesse auf monetärerer $\mathrm{Ba}$ sis an Bedeutung gewinnen (Cachay et al. 2001).

2. Weitere Gründe werden in den Alternativen zur Vereinsmitgliedschaft gesehen. Demnach optimieren Mitglieder ihr Verhalten entsprechend den damit verbundenen direkten Kosten, Zeitinvestitionen und Opportunitätskosten. Und mit zunehmenden Freizeit- bzw. Beschäftigungsalternativen steigen die Opportunitätskosten einer Vereinsmitgliedschaft (z. B. Heinemann, 1995; Schlagenauf, 1977). Demzufolge halten zweckorientierte Mitglieder ihre Investitionen zeitlicher und finanzieller Ressourcen in Sportvereine nur solange aufrecht, wie der in Aussicht gestellte Nutzen einer Vereinsmitgliedschaft - im Sinne der Befriedigung ihrer sportbezogenen und sozialen Interessen - die anfallenden Kosten für die Ressourcenabgabe übersteigt (Braun \& Nagel, 2005; Emrich et al. 2001).

3. Darüber hinaus werden instabile Mitgliedschaftsverhältnisse im Zusammenhang mit intraorganisationalen Veränderungsprozessen gesehen (z. B. Einrichtung neuer Angebotsund Abteilungsstrukturen, die Anstellung hauptberuflicher Mitarbeiter), indem der Aufbau kollektiv geteilter Handlungsorientierungen, die als Basis stabiler Mitgliedschaftsverhältnisse in einer Freiwilligenorganisation gel- ten, nicht mehr gelingt. Insbesondere die Wachstumsbestrebungen von Sportvereinen und die damit verbundene Öffnung für Nichtmitglieder erhöhen die Wahrscheinlichkeit einer Divergenz zwischen individuellen und kollektiven Interessen (z. B. Emrich, 2009; Klenk, 2011), was mit einer Aufweichung der Bindungsverhältnisse zwischen Mitglied und Sportverein einhergeht (z. B. Cachay, 1988; Nagel, 2006a).

Aktuelle Befunde der Sportvereinsforschung zeigen indes, dass nicht alle Vereine in gleichem Maße mit instabilen Mitgliedschaftsverhältnissen konfrontiert werden. So gibt es mit Blick auf die Mitgliederstruktur durchaus Vereine, die - aufgrund ihrer spezifischen situativen und strukturellen Bedingungen - kaum Probleme mit Mitgliederfluktuation und Vereinsaustritten haben, wohingegen andere Vereine mitunter erhebliche Mitgliederrückgänge verzeichnen (Lamprecht et al. 2012; s. auch Timm, 1979). Demnach ist zu vermuten, dass sich das soziale Handeln der Vereinsmitglieder je nach Organisationsprofil der Vereine (z. B. der Vereinsgröße, der inhaltlichen Ausrichtung) unterscheidet. Für die Frage der Mitgliederbindung scheinen deshalb sowohl die vereinsspezifischen Gegebenheiten als auch die individuellen Interessen, Erwartungen und Wertorientierungen der Mitglieder von zentraler Bedeutung zu sein. Im Gegensatz zu bislang vorliegenden Untersuchungen zur Mitgliedschaft in Sportvereinen, die Vereinsmitgliedschaften analytisch getrennt auf der Mitgliederebene anhand von Mitgliederbefragungen (z. B. Baur \& Braun, 2001; Nagel, 2006a, b) 


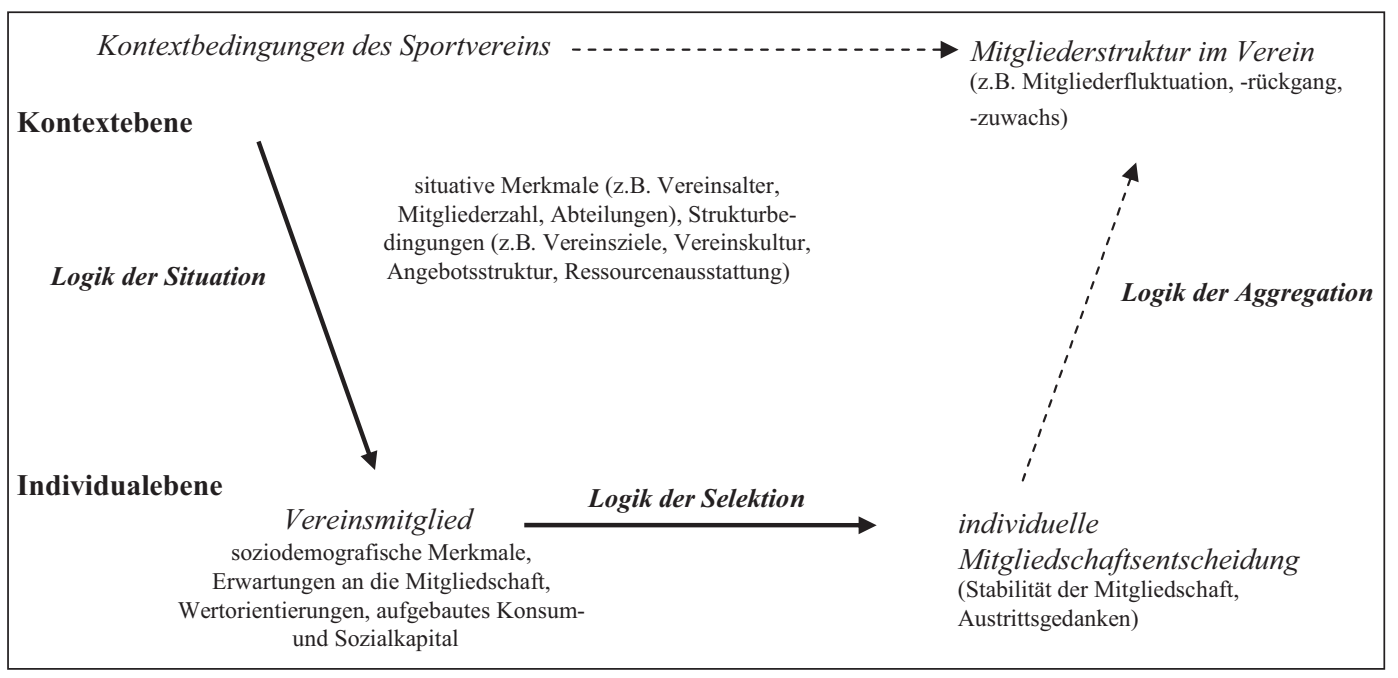

Abb. 1 Mehrebenenmodell zur Analyse der Mitgliederbindung im Sportverein oder auf der Vereinsebene anhand aggregierter vereinsbezogener Strukturdaten (Breuer, 2009, 2011; Lamprecht et al. 2012) untersuchen, sind auf Grundlage einer mehrebenenanalytischen Betrachtungsweise sowohl individuelle als auch kontextuelle Bedingungsfaktoren der Mitgliederbindung zu berücksichtigen. Zwar werden Verknüpfungen von Individual- und korrespondierenden Strukturdaten innerhalb der Sportvereinsforschung bereits seit geraumer Zeit gefordert (z. B. Emrich, Papathanassiou \& Pitsch, 1999; Nagel, 2007), aber bis heute nicht konsequent umgesetzt. Das Ziel der folgenden Analyse besteht darin, mögliche überindividuelle Faktoren der Mitgliederbindung im organisierten Sport zu identifizieren, indem individuelles Entscheidungsverhalten sowohl durch persönliche Merkmale als auch durch den sozialen Handlungskontext Sportverein strukturiert wird. Es stellt sich deshalb die Frage, welche organisations- und individuumsbezogenen Faktoren für die Mitgliederbindung in Sportvereinen eine Rolle spielen. Welche Determinanten beeinflussen die Wahrscheinlichkeit für den Verbleib in einem Sportverein oder die Entscheidung für den Austritt?

Zur Analyse der Bedingungen langfristiger Mitgliedschaften in Sportvereinen wird wie folgt vorgegangen: Zunächst werden relevante kontextuelle und individuelle Bestimmungsgründe der Mitgliedschaftsbindung in Sportvereinen diskutiert und zu Annahmen bzw. Fragestellungen verdichtet. In einem weiteren Schritt wird das methodische Vorgehen der empirischen Mehrebenenanalyse dargestellt, bevor die verschiedenen Determinanten einer systematischen Überprüfung in unterschiedlichen Modellschätzungen unterzogen werden. Eine Diskussion der Befunde schließt den Beitrag ab.

\section{Theoretische Überlegungen}

Im Zusammenhang mit der Frage der Mitgliederbindung wird davon ausgegangen, dass kontextuelle Bedingungen individuelle Entscheidungen und Verhaltensweisen strukturieren und persönliche Handlungsketten beeinflussen können (grundlegend dazu Coleman, 1990; Esser, 1999). Vor diesem Hintergrund wird der organisationale Kontext Sportverein nachfolgend als Interessenorganisation konzeptualisiert, der mit seinen Kontexteigenschaften als Gelegenheits- und Opportunitätsstruktur (z. B. Möglichkeiten des gemeinsamen Sporttreibens), als kultureller Bezugsrahmen (z. B. Werte, Traditionen) sowie als sozialer Bezugsrahmen (z. B. Identifikation, Freundschaften) gewisse Anreize schafft („Logik der Situation“), die gemäß individueller Präferenzen („Logik der Selektion“) zu Parametern des Mitgliederhandelns werden können (Büschges \& Abraham, 1997; Nagel, 2006a; • Abb. 1).

Sportvereine sind als Interessenorganisation den Bedürfnissen der Mitglieder verpflichtet (Horch, 1992). Das heißt, kollektives Handeln im Sportverein ist dadurch gekennzeichnet, dass die Mitglieder ihre individuellen Ressourcen zur Verwirklichung gemeinsamer Interessen bündeln (Coleman, 1974; Vanberg, 1978).
Der Vorteil dieser Ressourcenbündelung liegt darin, dass die Vereinsmitglieder ihre sportbezogenen Eigeninteressen mit Hilfe anderer Akteure in arbeitsteiliger Weise weitaus effektiver und effizienter erreichen können, als dies als Einzelakteur möglich wäre (Heinemann, 2004). Dazu bringt der Einzelne entsprechende Ressourcen in einen Pool ein, um das übergeordnete Ziel, die Produktion von Klubgütern (z. B. Sportangebote) gemeinsam zu verwirklichen, von denen sie wiederum selbst, aber auch andere Mitglieder durch Konsum profitieren können. Mit diesem „innenorientierten konsumatorischen Ethos“ (Schimank, 2005, S. 26) stellen Sportvereine ein klares Gegenbild zu anderen vorherrschenden Organisationstypen dar. In Sportvereinen gibt es somit nicht die etwa für Arbeitsorganisationen charakteristische Zweck-Motiv-Trennung, sondern die Bedienung dieser Mitgliedermotive bildet den konstitutiven Organisationszweck. Der Verfolgung dieses Zwecks zuwiderlaufende Strukturbedingungen von Vereinen sind demnach als destabilisierende Faktoren zu begreifen, sofern dadurch die Sinnhaftigkeit der Mitgliedschaft in einer Interessenorganisation gefährdet wird. Dementsprechend sind im Folgenden die Zusammensetzung des organisationalen Kontextes, die inhaltliche Ausrichtung, die Ressourcenausstattung sowie geografische Bedingungen im Zusammenhang mit der dauerhaften Mitgliedschaft im Verein zu diskutieren. 


\section{Strukturelle Determinanten}

Zunächst kann die Art der Zusammensetzung des organisationalen Kontextes für die Stabilität von Vereinsmitgliedschaften ausschlaggebend sein, wobei die empirische Befundlage diesbezüglich ambivalent ist. Mit zunehmender Vereinsgröße und Abteilungsdifferenzierung (und damit Ausdifferenzierung des Sportangebots) werden vielfältigere und heterogene Interessenansprüche von Seiten der Mitglieder an die Vereine herangetragen. Im Zusammenhang mit der Erstellung von Klubgütern wird durch die Organisationsgröße zugleich das Verhältnis von Ressourceneinbringung und erwartetem Nutzen gesteuert (z. B. Sandler \& Tschirhart, 1980). Denn innerhalb des Sportvereins produzieren Mitglieder als ehrenamtlich Tätige Klubgüter, deren Nutzen für ein Individuum sinkt, je mehr Individuen an diesem Gut partizipieren. Somit ist für jeden Verein eine optimale Organisationsgröße anzunehmen, bei der die einzelnen Organisationsmitglieder aus ihren eingebrachten Ressourcen zur Klubguterstellung die maximale Befriedigung ihrer Interessen ziehen können. Ab einer bestimmten Größe der am Klubgut partizipierenden Gruppe ist der Nutzenzuwachs für das Individuum geringer als die zusätzlich von ihm zu tragenden Produktionskosten. Hingegen steigt mit der Breite des Angebots innerhalb eines Vereins die Wahrscheinlichkeit der individuellen Befriedigung, da den unterschiedlichen Interessen und Präferenzen der Mitglieder zahlreiche Auswahlmöglichkeiten gegenüberstehen.

Darüber hinaus wird eine intensivere Bindung der Mitglieder und solidarisches Handeln eher bei kleinen als bei den großen Vereinen vermutet. Dies wird damit begründet, dass bei kleineren Vereinen der Gemeinschaftskern weitgehend alle Mitglieder umfasst, was wiederum das gemeinschaftliche Handeln fördert (Strob, 1999). Empirische Befunde machen jedoch deutlich, dass auch größere Vereine mit vielen Abteilungen durchaus eine hohe Verbundenheit ihrer Mitglieder aufweisen (Braun, 2003). Dies dürfte damit zusammenhängen, dass Mitglieder soziale Bezüge weniger zum Gesamtverein entwickeln, sondern die jeweilige Sportgruppe (z. B. Frauengymnastik)

Sportwiss 2013 · 43:90-101 DOI 10.1007/s12662-013-0291-y

(c) Springer-Verlag Berlin Heidelberg 2013

T. Schlesinger $\cdot$ S. Nagel

\section{Individuelle und strukturelle Faktoren der Mitgliederbindung im Sportverein}

\section{Zusammenfassung}

In diesem Beitrag werden Bedingungen analysiert, die die Mitgliederbindung in Sportvereinen beeinflussen. Neben individuellen Merkmalen interessieren dabei auch die Strukturbedingungen der Sportvereine, die im Zusammenhang mit der individuellen Wahlhandlung zwischen stabiler Mitgliedschaft oder Austritt stehen. Der Einfluss der Individual- und Kontextebene auf die Mitgliederbindung in Sportvereinen wird anhand unterschiedlicher Mehrebenenmodelle geschätzt. Die Analysen machen deutlich, dass sich die dauerhafte Mitgliedschaft in Sportvereinen nicht allein auf individuelle Merkmale der Mitgliedschaft, wie eine ausgeprägte Verbundenheit, ein positiv wahrgenommenes soziales Miteinander, die Zu- friedenheit mit der Vereinsarbeit sowie die ehrenamtliche Mitarbeit zurückführen lässt. Darüber hinaus nehmen vereinsspezifische Strukturbedingungen Einfluss auf die Mitgliederbindung, wobei in ländlich geprägten Sportvereinen und in Vereinen, die Geselligkeit explizit fördern und in denen das Vereinsziel sportlicher Erfolg eher eine untergeordnete Rolle spielt, die Austrittswahrscheinlichkeit geringer ist.

\section{Schlüsselwörter}

Sportvereine - Mitgliedschaft . Interessenorganisation · Mitgliederbindung . Mehrebenenanalyse

\section{Individual and structural factors of member commitment in sports clubs}

\section{Abstract}

This article analyses the conditions influencing the commitment of members of sports clubs. It focuses not only on individual characteristics of members, but also on the corresponding structural conditions of sports clubs related to the individual decision to quit or continue their membership. The influences of both the individual and context levels on the commitment of members are estimated in different multi-level models. Results of these multi-level analyses indicate that commitment of members is not just an outcome of individual characteristics such as strong commitment to the club, positive- ly perceived communication and cooperation, satisfaction with sports clubs' offers, or voluntary engagement. It is also influenced by club-specific structural conditions: commitment is more probable in rural sports clubs, and clubs who explicitly support sociability, whereas success-oriented sporting goals in clubs have a destabilizing effect.

\section{Keywords}

Sports clubs · Membership · Interest organization - Commitment of members . Multi level analysis oder Abteilung, die quasi eigene Gemeinschaften innerhalb des Vereins darstellen. So ist davon auszugehen, dass sich unter dem organisatorischen Dach eines Großvereins kleinere Wahlgemeinschaften zusammenschließen, in denen jeweils spezifische personenbezogene Netzwerke entstehen, die für die Mitgliedschaftsbeziehung förderlich sind (Braun, 2003; Nagel, 2006a). Zusammenfassend betrachtet stellt sich die Frage, inwiefern die Vereinsgröße und Abteilungsdifferenzierung die Mitgliederbindung beeinflussen.

Zur Vertretung und Realisierung der Mitgliederinteressen sind die inhaltliche
Ausrichtung und Ziele des Vereins maßgebend. Hierbei wird in der sportsoziologischen Diskussion vielfach zwischen zwei (analytischen und polarisierenden) Idealtypen differenziert: dem solidargemeinschaftlichen und dem dienstleistungsorientierten Sportvereintypus (z. B. Baur \& Braun, 2001; Emrich et al. 2001), was sich entsprechend auf die sozialen Beziehungsmuster und Handlungsorientierungen der Mitglieder auswirkt. In der Realität lassen sich diese Idealtypen zwar so nicht finden, vielmehr kombinieren Sportvereine verschiedene Leistungsbündel und Ziele miteinan- 
der, so dass Sportvereinstypologien einer wesentlich größeren Differenziertheit unterliegen (Nagel, 2006a). Gleichwohl ist davon auszugehen, dass Sportvereine, für die ein breites Angebot, Offenheit für neue Entwicklungen und Nichtmitglieder, Verberuflichungs- und Kooperationsbestrebungen wichtige Vereinsziele darstellen, in stärkeren Maße dienstleistungsorientiert sind, während die Förderung von Tradition und Geselligkeit eher dem solidargmeinschaftlichen Vereinstypus zu zuschreiben sind (Nagel et al. 2004, S. 49). In diesem Zusammenhang ist zu vermuten, dass die Mitglieder unterschiedliche Handlungsorientierungen und Entscheidungslogiken entwickeln (Braun \& Nagel, 2005): Solidargemeinschaftliche Vereine sind eher durch tradierte, wertrationale Handlungsorientierungen und stabilere Mitgliedschaftsbeziehungen gekennzeichnet, wobei die Sportvereinskultur und soziale Bindungen eine zentrale Rolle für die Mitgliedschaftsentscheidung spielen. Hingegen zeichnen sich dienstleistungsorientierte Vereine eher durch zweckrationale Handlungsorientierungen und hybride Mitgliedschaftsbeziehungen aus, wobei sich die Mitgliedschaftsentscheidungen stärker durch die Gegenüberstellung von Anreizen und Beiträgen vollziehen. Dementsprechend wird davon ausgegangen, dass traditionell und solidargemeinschaftlich ausgerichtete Vereine durch stabilere Mitgliedschaftsbeziehungen gekennzeichnet sind.

Die Frage nach der Stabilität einer Mitgliedschaftsbeziehung ist zudem eng verknüpft mit den zur Verfügung stehenden Alternativangeboten, die zur Befriedung individueller Interessen herangezogen werden können. Dabei spielen die geografische Lage bzw. die siedlungsstrukturellen Gegebenheiten, in denen ein Sportverein eingebettet ist, eine zentrale Rolle. In urbanen Räumen stehen Vereinsmitgliedern vielfältigere und ausdifferenzierte Alternativen der Freizeitgestaltung (Opportunitätsstrukturen) zur Verfügung als in ländlichen Regionen. Breitere Möglichkeiten der Freizeitgestaltung erhöhen damit die Opportunitätskosten von Vereinsmitgliedschaften (Schlagenauf, 1977). In ländlichen Regionen ist die Wahrscheinlichkeit, im Falle eines Austritts eine pas- sende Alternative zu finden, hingegen als geringer einzuschätzen. Denn bezogen auf begrenzte geografische Einzugsbereiche sowie bestimmte Sportformate und -arten können Sportvereine durchaus noch eine Monopolstellung einnehmen. Gleichzeitig schaffen bevölkerungsdichte Gebiete durch die potenzielle Erreichbarkeit anderer Menschen Gelegenheitsstrukturen, die organisiertes Handeln zwar erleichtern, dadurch aber wiederum Austritte weniger kostspielig machen. Hingegen lassen Sportvereine in ländlich geprägten Gebieten - aufgrund ihres hohen Stellenwerts als soziale Institution und damit einhergehend eines höheren Vergemeinschaftungscharakters (Nagel, 2006a) einerseits sowie einer geringeren Anonymität und den damit verbundenen Sanktionsmöglichkeiten der Nichtmitgliedschaft (z. B. soziale Kosten) andererseits (Bühlmann \& Freitag, 2004) - stabilere Vereinsmitgliedschaften erwarten. Darüber hinaus ist die Dienstleistungsorientierung von Sportvereinen in Ballungsräumen aufgrund größerer Konkurrenz kommerzieller Sportanbieter mitunter stärker ausgeprägt (Nagel, 2006a). Demnach ist zu vermuten, dass ländlich geprägte Sportvereine über stabilere Mitgliedschaftsverhältnisse verfügen.

Die Leistungsfähigkeit von Sportvereinen in Bezug auf die Interessenbefriedigung ist zudem von der Ressourcenausstattung des Vereins abhängig. Aufgrund bestehender Ressourcenknappheiten können Vereine nicht alle Interessen gleichermaßen bedienen, so dass infolge von Verteilungsproblemen personale sowie interpersonale Interessendivergenzen (z. B. zwischen Abteilungen) entstehen können (z. B. Heinemann, 2004; Klenk, 2011). Darüber hinaus werden durch die begrenzte Ressourcenausstattung die Entwicklungsmöglichkeiten (z. B. Angebotserweiterungen) im Zuge veränderter Interessenslagen und Qualitätsansprüche limitiert (Nagel \& Schlesinger, 2012). Zwar sind Sportvereine durch den Effizienzvorteil der Ehrenamtlichkeit in der Lage, ihre Klubgüter vergleichsweise günstiger zu produzieren als andere Sportanbieter. Dennoch ist eine Vielzahl von Vereinen mit finanziellen Problemen oder einer abnehmenden Mitarbeitsbereitschaft konfrontiert, die eine Erhöhung der Eigenfinanzierung er- forderlich machen. Der Mitgliedsbeitrag stellt hierbei einen wirksamen Stellhebel dar. Zwar ist in Bezug auf die Sportnachfrage eher von einer unelastischen Nachfrage auszugehen, dennoch können zu hohe Beitragsstrukturen in Sportvereinen insbesondere dann mit elastischen Nachfragestrukturen einhergehen (Wicker, 2009), wenn individuelle Haushaltsbudgets begrenzt sind, das Preis-LeistungsVerhältnis von Vereinen als nicht mehr angemessen angesehen wird oder auf kostengünstigere Alternativen zurückgegriffen werden kann. Demnach ist davon auszugehen, dass sich höhere Beitragsstrukturen destabilisierend auf die Mitgliedschaft im Verein auswirken.

\section{Individuelle Determinanten}

Neben den Kontextbedingungen sind individuelle Merkmale für die Frage der Mitgliederbindung maßgebend. Die dazu vorliegenden Forschungsarbeiten führen eine Reihe bindungsrelevanter Faktoren an, die sich in mitgliedschaftsbezogene und soziodemografische Determinanten differenzieren lassen.

Die Mitgliedschaftsentscheidung in einer Interessenorganisation dürfte zunächst durch die mehr oder weniger große Übereinstimmung von Mitgliederinteressen und Vereinszielen beeinflusst werden. Die Befriedigung der Mitgliederinteressen hängt dabei mit den subjektiven Erwartungen und Bewertungen der Mitglieder hinsichtlich bestimmter Leistungen des Vereins zusammen. Dies lässt sich anhand der Gesamtzufriedenheit mit der Vereinsarbeit erfassen, die sich aus unterschiedlichen Teilaspekten (z. B. attraktives Sportangebot, Qualität der Sportanlagen und Trainer) gewichtet mit deren relativer Bedeutung zusammensetzt (Dürr, 2009; Nagel, 2006a). In der Sportvereinsforschung liegen mittlerweile mehrere Untersuchungen vor, die positive Wirkungen der Zufriedenheit auf die Mitgliederbindung aufzeigen (z. B. Gieß-Stüber \& Rücker, 2003; Nagel, 2006a; Woll, 2003). Demnach beschäftigen sich zufriedene Vereinsmitglieder seltener mit dem Vereinsaustritt, während unzufriedene Mitglieder häufiger über eine Aufkündigung der Mitgliedschaft nachdenken und daher eher austreten. 
In diesem Zusammenhang dürfte das sportspezifische Konsumkapital eine zentrale Rolle spielen, wonach der Nutzen, den ein Vereinsmitglied aus dem Konsum vereinsspezifischer Klubgüter zieht, abhängig ist von dem Wissensstand, den es sich über die Güter angeeignet hat (Stigler \& Becker, 1977). Je größer das Konsumkapital, desto größer ist auch der Nutzen, der aus der Mitgliedschaft im Verein gezogen wird. Hierbei ist davon auszugehen, dass dieses Konsumkapital bei Vereinsmitgliedern unterschiedlich stark ausgeprägt ist und insbesondere mit der Dauer und Intensität der Mitgliedschaft (z. B. Wettkampfaktivität, Kinder sind im eigenen Verein) zusammenhängt. Wer sich zudem im Verein ehrenamtlich engagiert, für den dürfte der Nutzen einer Vereinsmitgliedschaft entsprechend größer sein, da man unmittelbar am Prozess der Klubguterstellung beteiligt ist, von der man dann wiederum selbst profitiert (Schlesinger \& Nagel, 2011). In diesem Zusammenhang ist anzumerken, dass in Sportvereinen aufgrund demokratischer Entscheidungsstrukturen, die Mitglieder bei Leistungsverschlechterung oder Unzufriedenheit nicht nur die Option des Austritts haben, sondern sie können auch versuchen, die Situation zu verbessern. Neben Beschwerde oder Protest über das Stimmrecht, ist in Sportvereinen die Eigeninitiative der Mitglieder von Bedeutung (Braun \& Nagel, 2005). Die Bereitschaft zum Widerspruch (statt Austritt) steht dabei in Zusammenhang mit der Verbundenheit und Treue zu einer Organisation (Hirschman, 1974).

In Sportvereinen ist vermutlich nicht nur die Zufriedenheit mit bestimmten Leistungsfaktoren für die Mitgliedschaftsentscheidung ausschlaggebend, sondern es sind auch die kollektiv geteilten Handlungs- und Wertorientierungen sowie die emotionale und soziale Einbindung von Bedeutung (Strob, 1999; Braun \& Nagel, 2005). Zahlreiche Befunde aus der Commitment-Forschung belegen die Relevanz emotionaler und normativer Einbindung im Zusammenhang mit der Aufrechterhaltung von Mitgliedschaften in Organisationen (im Überblick Meyer et al. 2002; im Sportverein: Chen, 2004; Nagel, 2006a, b). Wer sich also mit dem Verein bzw. einzelnen strukturellen Einheiten (z. B. Abteilungen) verbunden fühlt und dort enge soziale Beziehungen pflegt, für den dürfte die Wahrscheinlichkeit des Vereinsaustritts eher gering sein. Demnach ist die Mitgliedschaftsentscheidung auch von vereinskulturellen Orientierungen abhängig, wobei eine gute Atmosphäre im Verein, ein positiv wahrgenommenes soziales Miteinander sowie eine hohe Verbundenheit zu beachten sind. In diesem Zusammenhang sind insbesondere bei langjährigen Vereinsmitgliedern, wettkampfsportlich aktiven Mitgliedern sowie ehrenamtlich tätigen Mitgliedern die Identifikation und das gemeinschaftliche Interesse stärker ausgeprägt (Nagel, 2006a). Je mehr Zeit jemand im Verein verbringt, desto eher avanciert ein Verein zu einem (unverzichtbaren) Lebensbestandteil und Identitätsträger, so dass andere Alternativen nicht (mehr) in Betracht gezogen werden. Dementsprechend ist davon auszugehen, dass sich eine hohe Verbundenheit mit dem Verein sowie ein positiv wahrgenommenes soziales Miteinander im Verein stabilisierend auf die Mitgliedschaftsbeziehung auswirken.

Arbeiten zur Frage der Mitgliedergewinnung zeigen, dass sozioökonomische Merkmale wie Alter, Geschlecht oder auch Bildung oder Nationalität (Migrationshintergrund) nach wie vor zentrale Prädiktoren der Sportvereinsmitgliedschaft darstellen. Mit Blick auf die Mitgliederbindung wird nun argumentiert, dass sich v. a. jüngere Vereinsmitglieder verstärkt durch hybride, zumindest aber flexiblere Mitgliedschaftsstrukturen (Vereinskarrieren) auszeichnen (Baur \& Burrmann, 2003). Jüngere Vereinsmitglieder sind offener für neue Sportarten, Bewegungs- und Freizeitaktivitäten. Gleichzeitig dürfte für junge Menschen eine breitere Angebotspalette existieren, während es für Ältere häufig nur wenige Sportmöglichkeiten gibt. Ein Vereinswechsel oder -austritt wird somit von Jüngeren vermutlich mit einer größeren Wahrscheinlichkeit als Chance für Neues gesehen, während Ältere eher an Bewährtem festhalten. Jedoch ist anzumerken, dass sich im Zuge der allgemeinen Sportentwicklung sowohl für ältere Menschen als auch für Frauen die sportive Angebotspalette in Vereinen deutlich vergrößert hat (Lamprecht et al. 2012; Nagel, 2006b). Vor diesem Hintergrund dürfte sich der Einfluss von Ge- schlecht und Alter auf die Austrittsentscheidung relativieren.

\section{Methode}

\section{Datenerhebung und Stichprobe}

Um gemäß dem Mehrebenenmodell den Zusammenhang von Vereinsstruktur und Mitgliederhandeln analysieren zu können, sind vereinsbezogene Strukturdaten und individuelle Mitgliederdaten in Beziehung zu setzen (z. B. anhand von Mehrebenenanalysen).

Hinsichtlich der Verknüpfung von Daten des korporativen Akteurs mit den Daten individueller Akteure liegt es deshalb nahe, Fallstudien bei ausgewählten Sportvereinen durchzuführen. Dementsprechend sind sowohl die sozialen Strukturen und situativen Rahmenbedingungen des Sportvereins als auch die Handlungsorientierungen und Erwartungen der Mitglieder zu erfassen. Eine Beschränkung auf wenige, aber für die gesamte Bandbreite der Schweizer Sportvereinslandschaft möglichst aussagekräftige Fälle, setzt eine kriteriengeleitete Auswahl der zu untersuchenden Sportvereine voraus. Als zentrale Arbeitsgrundlage für diesen Selektionsprozess diente die aktuelle Sportvereinsstudie in der Schweiz (Lamprecht et al. 2012). Folgende Kriterien waren bei der Auswahl maßgebend:

- Vereinsgröße (Mitgliederzahl, Abteilungszahl),

- Sportarten,

- Zielprofil,

- Ressourcenausstattung,

- Siedlungsstruktur,

- Sprachregion.

Als Datengrundlage für die Analyse diente eine Befragung in 45 ausgewählten Schweizer Sportvereinen, die eine hohe Bandbreite an unterschiedlichen Strukturtypen abdecken. ${ }^{1}$ Somit dürften die vorliegenden Befunde für den Vereinssport in der Schweiz weitgehend generalisierbar sein. In einem ers-

\footnotetext{
1 Die vorliegende Analyse ist Bestandteil des Projektes „Personale Ressourcen im Sportverein“ welches von der Eidgenössischen Sportkommission (ESK) und der Schweizerischen Gemeinnützigen Gesellschaft (SGG) finanziell unterstützt wurde.
} 
Tab. 1 Variablen zur Analyse der Mitgliederbindung und deren Operationalisierung

\section{Variable}

Individualfaktoren

Abhängige Variable: Maß der formalen

Mitgliederbindung (Austrittsrisiko)

Soziodemografische Faktoren

\section{Alter}

Alter quadriert

Mitgliedschaftsbezogene Faktoren ${ }^{\mathrm{a}}$

Kinder im Verein aktiv

(Globale) Mitgliederzufriedenheit

Mitgliedschaftsdauer

Wettkampfsportliche Aktivität

Verbundenheit

Soziales Miteinander

Ehrenamtliches Engagement

Kontextfaktoren

Situative Merkmale

Vereinsalter

Mitglieder

Abteilungen (Angebotsdifferenzierung)

Siedlungsstruktur

Ressourcenspezifische Merkmale

Ausstattung personale Ressourcen

\section{Beitragsstrukturen}

Haushaltsvolumen

\section{Ausrichtung/Ziele des Vereins ${ }^{c}$}

Erzielung von sportlichen Erfolgen

Engagement im Breitensport

Wahrung der Vereinstradition

Förderung von Geselligkeit

Offenheit für neue Entwicklungen

Kooperation mit anderen Einrichtungen

Verberuflichung

\section{Operationalisierung}

„Haben Sie in letzter Zeit darüber nachgedacht, die Mitgliedschaft in ihrem Verein zu beenden?" ( $1=$ nein bis $5=$ ja, häufig)

Dummy; 1 = männlich

Anzahl Lebensjahre (> 16 Jahre)

Quadrierte Anzahl Lebensjahre

Dummy; $1=$ Kinder im Verein ( $\leq 16$ Jahre)

$1=$ sehr unzufrieden bis $5=$ sehr zufrieden

Anzahl Mitgliedschaftsjahre

Dummy; $1=$ ja

Index aus 5 Items $^{\mathrm{b}}$

Index aus 4 Items ${ }^{b}$

Dummy; $1=$ ja

Vereinsalter in Jahren

Anzahl Mitglieder

Anzahl Abteilungen

1 = ländlich, 2 = Agglomeration, 3 = städtisch, 4= Großstadt

Verfügbarkeit Ressource "freiwillige Mitarbeit"

1 = keine Probleme; 3 = große Probleme

Durchschnittlicher Mitgliedsbeitrag pro Jahr für Erwachsene

$1=\leq 100$ CHF, $2=101-249$ CHF, $3=\geq 250$ CHF

Veranlagtes Haushaltsbudget pro Jahr in CHF

$1=\leq 25.000 \mathrm{CHF}, 2=25.001-49.999 \mathrm{CHF}, 3=50.000-99.999 \mathrm{CHF}, 4=\geq 100.000$

$1=$ nicht wichtig bis $5=$ wichtig

$1=$ nicht wichtig bis $5=$ wichtig

$1=$ nicht wichtig bis $5=$ wichtig

$1=$ nicht wichtig bis $5=$ wichtig

$1=$ nicht wichtig bis $5=$ wichtig

$1=$ nicht wichtig bis $5=$ wichtig

Dummy; 1 = bezahlte Mitarbeiter

alm Zuge der Prüfung auf Multikollinarität wurde der Faktor Aktivenjahre nicht in das Modell aufgenommen.

b(1) „Verbundenheit" (Index: M=4,08; SD =0,64; Cronbachs a =0,85) : Ich fühle mich wohl in unserem Verein. "Ich fühle mich mit meinem Verein verbunden. "ICh besuche gerne Veranstaltungen unseres Vereins." "Ich bin stolz, wenn ich anderen sagen kann, dass ich zum Verein gehöre." "Ich diskutiere mit anderen Mitgliedern über Vereinsangelegenheiten." (2) „soziales Miteinander" (Index: $M=4,16 ; S D=0,66 ;$ Cronbachs $a=0,87$ ): "Wir pflegen einen offenen und freundschaftlichen Umgang."

„In unserem Verein herrscht eine gute Atmosphäre.“" "Über auftretende Probleme sprechen wir offen miteinander." "Wir legen viel Wert aufTeamgeist und partnerschaftliche Zusammenarbeit." Die Items wurden jeweils auf einer fünfstufigen Skala von $1=$ = trifft überhaupt nicht zu“ bis 5 "trifft voll und ganz zu“ erfasst

IIm Rahmen der Analyse wurde darauf verzichtet, die inhaltliche Ausrichtung von Vereinen anhand aggregierter Strukturprofile abzubilden. Stattdessen wurden die Ausprägungen einzelner Merkmale berücksichtigt. Diese wurden hinsichtlich ihrer Konsistenz geprüft: Die berechneten Korrelationen bezüglich der inhaltlichen Ausrichtung lassen keine Inkonsistenzen erkennen.

ten Schritt wurden verschiedene strukturelle Merkmale der ausgewählten Sportvereine über einen Strukturfragebogen (Beantwortung durch die Vereinsführung) erfasst. In einem weiteren Schritt wurden Mitgliederdaten via Onlinebefragung erhoben. Als Grundgesamtheit für die Mitgliederbefragung wurden alle Vereinsmitglieder herangezogen, die älter als 16 Jahre waren. Der überwiegende Teil der Schweizer Sportvereine weist (im Gegensatz zu Sportvereinen in Deutschland) eher geringe Mitgliederzahlen (<100 Mitglieder) auf und ist als Einspartenverein zu kennzeichnen, so dass dementsprechend Dienstleistungsorientierung und Verberuflichung weniger ausgeprägt sind. Aufgrund der eher geringen Mitgliederzahlen wurde auf die Ziehung von einfachen Zufallsstichproben verzichtet und 
es wurde jeweils eine Vollerhebung angestrebt. In den ausgewählten Sportvereinen wurden die Vereinsmitglieder mit gültiger E-Mail-Adresse über die Befragung entsprechend informiert und zur Teilnahme aufgerufen. Vereine mit unvollständig gepflegten E-Mail-Adressen hatten zudem alternativ die Möglichkeit, den Fragebogen in ausgedruckter Version an ihre Mitglieder zu verteilen. Ein Nachteil von Online-Befragungen ist, dass meist nur Personen mit einem bestimmten Mediennutzungsverhalten befragt werden, so dass eine Einflussnahme auf die Repräsentativität hinsichtlich Alter und Geschlecht nur beschränkt möglich ist. Dieses Problem der Stichprobenselektivität dürfte für die vorliegende Analyse jedoch eher gering sein, da in den meisten untersuchten Sportvereinen ein Großteil der Mitglieder per Mail erreichbar war. Nach der Datenbereinigung konnte in der Hauptstudie ein Sample von $n=1528$ befragten Sportvereinsmitgliedern generiert werden. Von den befragten Mitgliedern sind 39,3\% weiblich und $60,7 \%$ männlich. Die ungleiche Geschlechterverteilung ist darauf zurückzuführen, dass Frauen in Schweizer Sportvereinen nach wie vor unterrepräsentiert sind (etwa $36 \%$ der Sportvereinsmitglieder sind weiblich; Lamprecht et al. 2012). Bezüglich der Altersverteilung ist zu beachten, dass in unserer Studie nur Vereinsmitglieder über 16 Jahre befragt wurden, so dass Abweichungen zur Grundgesamtheit bestehen. Die Altersverteilung ( $M=36,2$ Jahre; $S D \pm 15,9)$ stellt sich wie folgt dar: $17,6 \%$ bis 20 Jahre; 45,3\% 21-40 Jahre; 28,1\% 41-60 Jahre und 9,0 \% über 61 Jahre). Die Mitgliedschaftsdauer ( $M=14,8$ Jahre; $S D \pm 12,6)$ verteilt sich wie folgt: $30,4 \%$ bis 5 Jahre; 16,5\% 6-10 Jahre; 25,3\% 11-20 Jahre; $27,8 \%$ über 21 Jahre. Darüber hinaus sind $65,2 \%$ der befragten Mitglieder in ihrem Verein ehrenamtlich tätig, 90,7\% sind derzeit Aktivmitglieder und 84,9\% der Vereinsmitglieder sind/waren wettkampfsportlich aktiv. ${ }^{2}$

\section{Operationalisierung}

Zur Operationalisierung des Austrittsrisikos (als Maß für die Mitgliederbindung) wurden die Vereinsmitglieder gefragt, inwieweit sie in den vergangenen Monaten (vor der Befragung) darüber nachgedacht haben, aus ihrem Verein auszutreten. $69,5 \%$ beantworteten diese Frage mit "nein", während sich 20,8\% „manchmal" und 11,7\% „öfters bzw. häufiger Gedanken" über einen Vereinsaustritt machen. Damit sind rund zwei Drittel der Mitglieder als „Vereinstreue " und etwa ein Drittel als „potenzielle Aussteiger“ zu charakterisieren. Zwar wird nicht jeder, der über einen Vereinsaustritt nachdenkt, auch tatsächlich die Mitgliedschaft kündigen, aber die Wahrscheinlichkeit für einen Vereinsaustritt dürfte bei den Mitgliedern, die bereits in einem entsprechenden Entscheidungsprozess eingetreten sind, deutlich höher sein. Die Operationalisierung der Konstrukte Mitgliederzufriedenheit, Verbundenheit sowie Kommunikation und soziales Miteinander orientierte sich an elaborierten Konzepten der Mitgliederforschung in Sportvereinen (z. B. Braun \& Nagel, 2005; Nagel, 2006a, b). Die Operationalisierung der strukturellen Vereinsmerkmale erfolgte in Anlehnung an gängige Verfahrensweisen aktueller Strukturanalysen (z. B. Breuer, 2009, 2011; Lamprecht et al. 2012; Nagel et al. 2004). Einen Überblick der zugrunde liegenden Individual- und Kontextfaktoren sowie deren Operationalisierung bietet $\mathbf{0}$ Tab. 1 .

\footnotetext{
2 Es ist zu vermuten, dass gemäß der Intention der Gesamtstudie, Ehrenamtliche und Wettkampfsportler in der Stichprobe überrepräsentiert sind. Eine mögliche Selektivität der Stichprobe liegt insbesondere darin begründet, dass mit der Studie keine repräsentative Beschreibung der Ehrenamtlichkeit bzw. der Mitgliederstruktur in Schweizer Sportvereinen angestrebt wurde, sondern es galt, spezifische Zusammenhänge und Einflussfaktoren bei individuellen Entscheidungsprozessen zu analysieren. Da jedoch auch für möglicherweise unterrepräsentierte Mitgliedergruppen hinreichend große Teilstichproben erfasst wurden, dürften die geprüften Annahmen für alle Vereinsmitglieder gültig sein.
}

\section{Datenanalyse}

Die adäquate statistische Methode, welches Determinanten der Mitgliederbindung auf beiden Ebenen (Mitglied und Sportverein) berücksichtigen kann und folglich das entwickelte theoretische Modell statistisch umsetzt, ist die Mehrebenenanalyse (Hox, 2002). Diese Analysemethode gewährleistet robuste Befunde bei simulierten Schätzungen von Individualdaten und korrespondierenden Strukturdaten. Denn weder eine reine Aggregat- noch eine reine Individualdatenanalyse liefern i. d. R. befriedigende Resultate. Bei Aggregatdaten setzt man sich der Gefahr des Fehlschlusses und der Kritik der Vernachlässigung der originären Datenstruktur aus, während die reine Individualdatenanalyse der inhärenten hierarchischen Strukturen nicht gerecht wird (Raudenbush \& Bryk, 2002). Hingegen verdeutlichen Mehrebenenanalysen die Effekte zwischen Variablen auf mehreren Ebenen und identifizieren somit Zusammenhänge, die bei herkömmlichen Regressionsanalysen unentdeckt bleiben (Hox, 2002). Erst durch die Modellierung der hierarchischen Struktur der Daten (jede Messung auf der Individualebene kann eindeutig einer Messung auf der Vereinsebene zugeordnet werden) lässt sich die Wirkung individueller und kontextueller Merkmale bestimmen. Mit Hilfe der Mehrebenenanalyse wird also geschätzt, ob und zu welchem Anteil sich die Varianz einer stabilen Vereinsmitgliedschaft (bzw. das Austrittsrisiko) sowohl auf Unterschiede zwischen den Mitgliedern als auch auf Unterschiede zwischen den Vereinen zurückführen lässt. Dementsprechend wird die Varianz zwischen den Mitgliedern durch individuelle Merkmale und die Varianz zwischen den Kontexten (Vereinen) durch Strukturmerkmale zu erklären versucht. Da die zu erklärende Variable "Mitgliederbindung“ ein näherungsweise metrisches Skalenniveau aufweist, wurde eine OLS-basierte Mehrebenenmethode (OLS: "ordinary least squares") verwendet.

Das Verfahren der Mehrebenenanalyse stellt einige Anforderungen an die Datenstruktur. Um statistisch gehaltvolle Aussagen treffen zu können, werden hierarchische Erhebungen benötigt, die für je- 
Tab. 2 Individuelle und kontextuelle Determinanten der Mitgliederbindung (nicht standardisierte Koeffizienten)

\begin{tabular}{|c|c|c|c|c|c|c|}
\hline & Modell 0 & Modell 1 & Modell 2 & Modell 3 & Modell 4 & Modell 5 \\
\hline \multicolumn{7}{|l|}{ Fixed part } \\
\hline Konstante (Intercept) & $1,598^{* * *}$ & $4,029^{* * *}$ & $3,564^{* * *}$ & $3,368^{* * *}$ & $3,852^{* * *}$ & $3,205^{* * *}$ \\
\hline \multicolumn{7}{|l|}{$\begin{array}{l}\text { Individualfaktoren der } \\
\text { Mitgliederebene }\end{array}$} \\
\hline \multicolumn{7}{|l|}{ Soziodemografische Merkmale } \\
\hline Geschlecht (1 = männlich) & & 0,070 & 0,070 & 0,074 & 0,077 & 0,085 \\
\hline Alter & & 0,002 & 0,002 & 0,004 & 0,006 & 0,006 \\
\hline Alter quadriert & & 0,000 & 0,000 & 0,000 & 0,000 & 0,000 \\
\hline \multicolumn{7}{|l|}{ Mitgliedschaftsbezogene Merkmale } \\
\hline Kinder im Verein aktiv $(1=\mathrm{ja})$ & & $-0,092$ & $-0,088$ & $-0,093$ & $-0,098$ & $-0,083$ \\
\hline (Globale) Mitgliederzufriedenheit & & $-0,101^{* *}$ & $-0,101^{* *}$ & $-0,097^{* *}$ & $-0,094^{* *}$ & $-0,100^{* *}$ \\
\hline Mitgliedschaftsdauer & & $-0,002$ & $-0,004$ & $-0,003$ & $-0,003$ & $-0,004$ \\
\hline Wettkampfsportliche Aktivität $(1=\mathrm{ja})$ & & 0,014 & 0,013 & 0,014 & 0,027 & 0,027 \\
\hline Verbundenheit & & $-0,286^{* * *}$ & $-0,282^{* * *}$ & $-0,294^{* * *}$ & $-0,305^{* * *}$ & $-0,284^{* * *}$ \\
\hline Soziales Miteinander & & $-0,200^{* * *}$ & $-0,206^{* * *}$ & $-0,188^{* * *}$ & $-0,166^{* * *}$ & $-0,185^{* * *}$ \\
\hline Ehrenamtliches Engagement $(1=\mathrm{ja})$ & & $-0,153^{*}$ & $-0,156^{*}$ & $-0,169^{*}$ & $-0,165^{*}$ & $-0,165^{*}$ \\
\hline \multicolumn{7}{|l|}{ Strukturfaktoren der Vereinsebene } \\
\hline \multicolumn{7}{|l|}{ Situative Merkmale } \\
\hline Vereinsalter & & & $-0,001$ & - & - & - \\
\hline Anzahl Mitglieder & & & 0,004 & - & - & - \\
\hline Anzahl Abteilungen & & & $-0,019$ & - & - & - \\
\hline Siedlungsstruktur & & & $0,164^{*}$ & - & - & $0,145^{*}$ \\
\hline \multicolumn{7}{|l|}{ Ressourcenspezifische Merkmale } \\
\hline Haushaltsvolumen pro Jahr & & & - & 0,089 & - & - \\
\hline Beitragsstrukturen pro Jahr & & & - & $0,167^{*}$ & - & 0,162 \\
\hline Ausstattung personale Ressourcen & & & - & 0,007 & - & - \\
\hline \multicolumn{7}{|l|}{ Ausrichtung/Ziele des Vereins } \\
\hline Erzielung von sportlichen Erfolgen & & & - & - & $0,143^{*}$ & $0,166^{*}$ \\
\hline Engagement im Breitensport & & & - & - & $-0,023$ & - \\
\hline Wahrung der Vereinstradition & & & - & - & 0,037 & - \\
\hline Förderung von Geselligkeit & & & - & - & $-0,197^{*}$ & $-0,152^{*}$ \\
\hline Offenheit für neue Entwicklungen & & & - & - & $-0,036$ & - \\
\hline Kooperation mit anderen Einrichtungen & & & - & - & 0,024 & - \\
\hline Verberuflichung ( $1=$ bez. Mitarbeit) & & & - & - & $-0,045$ & - \\
\hline \multicolumn{7}{|l|}{ Random part } \\
\hline Individualvarianz $\left(\sigma^{2}\right)$ & 1,193 & 0,989 & 0,985 & 0,990 & 0,986 & 0,973 \\
\hline Kontextvarianz $\left(\mathrm{\tau}_{00}\right)$ & 0,189 & 0,123 & 0,098 & 0,097 & 0,101 & 0,072 \\
\hline ICC $(\rho)$ & 0,137 & 0,110 & 0,090 & 0,089 & 0,093 & 0,069 \\
\hline Devianz (2-log Likelihood) & 4387,2 & 2895,6 & 2905,4 & 2885,9 & 2871,1 & 2866,7 \\
\hline
\end{tabular}

den Verein eine Mindestzahl an Befragten aufweisen. In der einschlägigen Literatur besteht allerdings kein Konsens hinsichtlich der Anzahl der Fälle auf der Strukturebene. Grundsätzlich ist davon auszugehen, dass die Modellstärke mit einer steigenden Fallzahl auf dem höheren Level zunimmt (Hox, 2002). Entsprechend werden i. d. R. mindestens 25 Fälle auf dem höchsten Level (hier Vereins- ebene) und jeweils mindestens 20 Beobachtungen innerhalb jeder tieferliegenden Ebene (Mitgliederebene) empfohlen (Hox, 2002; Snijders \& Bosker, 1999). Demzufolge wurden in unserer Studie alle Sportvereine, die weniger als 20 Beobachtungen (befragte Mitglieder) enthalten, aus der Analyse ausgeschlossen. Somit konnten 36 Sportvereine ( $n=1434$ Mitglieder) für die weitere Analyse berück- sichtigt werden. Die Datenanalyse erfolgte mit SPSS Version 20 (gemischte Modelle). Aufgrund der eher geringen Anzahl von Fällen auf Level-2-Ebene wurde im Rahmen der Analyse das eingeschränkte Maximum-Likelihood (REML) als Schätzverfahren verwendet.

Im Rahmen der Datenanalyse wird wie folgt vorgegangen: 1) Um zunächst die Varianz der abhängigen Variablen auf indi- 
vidueller und kontextueller Ebene zu dokumentieren, wird ein Nullmodell (Random-intercept-only-Modell) geschätzt. Dabei dient die Höhe des Intraklassenkorrelationskoeffizienten (ICC) als Entscheidungskriterium, ob eine Mehrebenenanalyse die adäquate Analysestrategie darstellt: Ließe sich keine Varianz der abhängigen Variablen auf der höheren Ebene ausmachen (ICC hat einen Wert nahe 0 ), so erübrigte sich auch die Erklärung der Varianz durch Strukturmerkmale der Vereine, und eine Mehrebenenanalyse wäre nicht angemessen (Hox, 2002). 2) Daran schließt sich die Schätzung der Individualmerkmale an, wobei die potenziellen Strukturunterschiede kontrolliert bleiben. 3) Um den Einfluss individueller und kontextueller Bedingungen auf die Stabilität der Mitgliedschaft simultan zu prüfen, wird eine Reihe von Mehrebenenmodellen durchgeführt. Diese Modelle beinhalten die Individualmerkmale, unterscheiden sich aber durch die herangezogenen Kontextgrößen. Für das weitere Vorgehen wird empfohlen, nicht alle diskutierten Kontextgrößen gleichzeitig in ein Modell einfließen zu lassen (Bühlmann \& Freitag, 2004). Dementsprechend werden lediglich einzelne Variablenbündel getestet: Das Modell 2 schätzt den Einfluss situativer Merkmale, Modell 3 schätzt den Einfluss ressourcenspezifischer Bedingungen, während Modell 4 die inhaltliche Ausrichtung des Vereins berücksichtigt. Die sich in diesen Schätzungen als signifikant erweisenden Kontextgrößen sind anschließend in ein kombiniertes Gesamtmodell zu integrieren (Modell 5).

\section{Ergebnisse}

Die Ergebnisse der verschiedenen Modellschätzungen sind in $\boldsymbol{0}$ Tab. 2 dargestellt. Als übergeordnete Vermutung wird zunächst angenommen, dass die Stabilität der Mitgliedschaft in Sportvereinen nicht nur zwischen Individuen, sondern auch zwischen Strukturkontexten der Vereine variiert. Deshalb wurde vorgängig ein Nullmodell berechnet, das die Varianzanteile der beiden Ebenen (Individual- und Strukturebene) schätzt. Es handelt sich hier also um ein nicht spezifiziertes Modell, in welchem keine Variablen, sondern lediglich der Effekt der Kons- tanten (Gruppierungsvariable) modelliert wird. Im Nullmodell (• Tab.2, erste Spalte) beträgt die Varianz der Residuen auf der Individualebene (Mitglieder) $\sigma^{2}=1,19$; die Kontextvarianz beträgt $\tau_{00}=0,19$. anschließend erfolgte die Berechnung des Intraklassenkorrelationskoeffizienten. Es ergibt sich eine Intraklassenkorrelation von $\rho=0,14$. Der ICC gibt dabei den Anteil der Level-2-Varianz an der Gesamtvarianz in der abhängigen Variablen wieder. Damit zeigt sich, dass etwa $14 \%$ der Varianz der Mitgliederbindung auf Unterschiede zwischen den Vereinen zurückgehen. Die Schätzung des Nullmodells bestätigt somit die Ausgangsvermutung, so dass eine simultane Schätzung von Individual- und Strukturmerkmalen mittels einer Mehrebenenanalyse auch in empirischer Hinsicht sinnvoll ist.

Folglich sind im nächsten Schritt die Faktoren der Mitgliederbindung zunächst auf der Individualebene zu schätzen. Das Random-intercept-Modell (Modell 1) dokumentiert die Schätzung aller Variablen auf der Individualebene, wobei die potenziellen Kontextunterschiede kontrolliert bleiben. Es zeigt sich ein signifikanter Einfluss von vier einbezogenen Variablen auf der Individualebene. Vor allem die Variablen Verbundenheit, ein als positiv wahrgenommenes soziales Miteinander, die (globale) Zufriedenheit mit dem Verein sowie ein ehrenamtliches Engagement fördern die Stabilität der Mitgliedschaft. Ohne signifikanten Einfluss sind die Variablen Alter, Geschlecht, Wettkampfaktivität, Mitgliedschaftsdauer sowie Kinder im Verein.

Um zu prüfen, welche strukturellen Bedingungen der Sportvereine die Mitgliederbindung beeinflussen, wurden im nächsten Schritt Schätzungen bezüglich der vereinsbezogenen Strukturmerkmale durchgeführt. Die Schätzungen der Random-intercept-Modelle 2-4 lassen zunächst folgende Schlüsse zu: Vier der insgesamt 14 einbezogenen Kontexteigenschaften von Sportvereinen weisen eine statistische Signifikanz auf und vermögen die kontextuellen Unterschiede der Mitgliederbindung zu erklären. Eher ländlich geprägte Sportvereine und eine hohe Relevanz des Vereinsziels „Förderung von Geselligkeit" reduzieren unabhängig von individuellen Merkmalen das Aus- trittsrisiko. Hingegen wirken sich höhere Mitgliedsbeiträge und eine hohe Bedeutung des Vereinsziels „Erzielung sportlicher Erfolge" eher destabilisierend auf die Mitgliederbindung aus. Keinen statistisch signifikanten Einfluss haben die Mitgliederzahl, die Anzahl an Abteilungen, das Vereinsalter, das Haushaltsvolumen, die personale Ressourcenausstattung, der Verberuflichungsgrad sowie die Vereinsziele Offenheit für neue Entwicklungen, Kooperation mit anderen Einrichtungen, Wahrung der Vereinstradition sowie Engagement im Breitensport. Werden die statistisch signifikanten Strukturvariablen in ein Random-intercept-Gesamtmodell überführt (Modell 5),verliert der Mitgliedsbeitrag allerdings an Erklärungskraft. Hingegen zeigt sich für die anderen drei Strukturfaktoren weiterhin ein statistisch signifikanter Einfluss auf die Mitgliederbindung: Ländlich geprägte Sportvereine sowie Vereine, die Geselligkeit explizit fördern, sportlichem Erfolg jedoch eine nicht allzu große Bedeutung beimessen, zeichnen sich durch stabilere Mitgliedschaftsverhältnisse aus. ${ }^{3}$

Hinsichtlich der Modellgüte ist Folgendes festzuhalten: Zunächst wird deutlich, dass sich in den verschiedenen Modellen die Schätzwerte der Individualund Kontextvarianz verändern. Die Intraklassenkorrelation geht insbesondere durch Berücksichtigung der Kontextebene zurück. Damit wird deutlich, dass die herangezogenen Strukturgrößen zur Aufklärung der Unterschiede zwischen den Vereinen beitragen. Des Weiteren lassen sich jeweils die Pseudo- $\mathrm{R}^{2}$-Werte für die Regressionen zwischen den Gruppen und innerhalb der Gruppen berechnen. Für die Veränderung gegenüber dem Nullmodell ergibt sich für Varianzkomponenten

3 Über die im Beitrag dokumentierten Schätzungen hinaus wurden noch weitere Modelle mit Interaktionen zwischen Kontext- und Individualebene (Cross-level-Interaktionen) berechnet. So zeigt sich z. B. ein signifikanter Einfluss der Kontextvariable Geselligkeit auf den Zusammenhang zwischen der subjektiv wahrgenommenen Kommunikation/Zusammenarbeit und der Mitgliederbindung. Damit wird deutlich, dass Kontexteigenschaften nicht nur direkt auf individuelles Handeln wirken, sie beeinflussen darüber hinaus - im Sinne der Logik der Situation - auch die Stärke des Zusammenhangs zwischen Akteur und dessen Handlungen. 
auf der Individualebene ein $R_{\text {within }}^{2}$ von 0,17 (in Modell 1). Die Varianzkomponenten auf der Kontextebene reduzieren sich ebenfalls. Im Vergleich zu Modell 1 erklären die vier Faktoren in Modell 5 die kontextuelle Varianz $\left(R_{\text {between }}^{2}\right) \mathrm{zu} 0,41 . \mathrm{Zu}$ beachten ist allerdings, dass der Wert aufzuklärender Kontextvarianz wesentlich kleiner ist als der Wert der Individualvarianz. Zur Beurteilung der Modellgüte kann zudem der Vergleich der DevianzWerte ( -2 log Likelihood) herangezogen werden. Der Wert für die - 2 log Likelihood geht von 4387,2 (Nullmodell) auf 2895,6 in Modell 1 (Individualdaten) und auf 2866,7 in Modell 5 (Individual- und Strukturdaten) zurück. Die Veränderung der Devianz zeigt an, dass sich durch die Berücksichtigung von Strukturgrößen die Schätzung insgesamt verbessert, das Modell also zu einem höheren Maß den empirischen Daten angepasst ist.

\section{Diskussion}

Es wurde von der Annahme ausgegangen, dass für die Mitgliederbindung nicht allein Individualmerkmale ausschlaggebend sind, sondern Sportvereine mit ihren kontextuellen Eigenschaften gewisse Rahmenbedingungen und Anreize bieten, die das Mitglied in seiner Mitgliedschaftsentscheidung direkt beeinflussen können. Hinsichtlich mitgliedschaftsbezogener Eigenschaften zeigt die Analyse, dass derjenige, welcher mit dem Verein stark verbunden ist, das Handeln im Verein als kommunikativ und kameradschaftlich wahrnimmt, mit der Vereinsarbeit weitgehend zufrieden ist und sich ehrenamtlich im Verein engagiert, ein geringes Austrittsrisiko aufweist. Damit bestätigen sich die Annahmen, dass für eine dauerhafte Mitgliedschaft sowohl zweckorientierte Nutzenüberlegungen (Zufriedenheit) als auch solidargemeinschaftliche Handlungsorientierungen, insbesondere die Verbundenheit, eine zentrale Rolle spielen, so dass eine ausschließliche Dienstleistungs- bzw. Kundenorientierung als Strategie der Mitgliederbindung in Sportvereinen, wie sie vielfach (auch von Verbänden) nahegelegt wird, zu kurz greifen dürfte. Diese Befunde sind konsistent mit bereits vorliegenden Befunden zur Mitgliederbindung in Sportvereinen (z. B. Nagel, 2006a, b). Hingegen sind die Faktoren Mitgliedschaftsdauer, Wettkampfaktivität, Kinder im Verein, Alter und Geschlecht ohne systematischen Einfluss auf die Mitgliederbindung. Insbesondere die These, dass jüngere Vereinsmitglieder ein höheres Austrittsrisiko aufweisen als ältere Vereinsmitglieder, kann anhand unserer Daten nicht bestätigt werden. Insgesamt wird deutlich, dass auf individueller Ebene ausschließlich mitgliedschaftsbezogene Faktoren die Mitgliederbindung beeinflussen, während Faktoren wie Alter oder Geschlecht - anders als bei Analysen der Mitgliedergewinnung - keinen nennenswerten Einfluss ausüben. Weiterhin zeigt sich, dass eine Aufnahme kontrollierender Kontextfaktoren die Resultate nur marginal verändert, was den robusten Einfluss der genannten individuellen Merkmale bestätigt.

Wie angenommen, bestehen Unterschiede bezüglich der Mitgliederbindung zwischen verschiedenen Vereinen. Die Intraklassenkorrelation zeigt, dass etwa $14 \%$ der Varianz der Mitgliederbindung auf strukturbedingte Unterschiede der Vereine zurückzuführen ist. Mit Blick auf die erklärungsrelevanten Kontexteigenschaften sind in ländlich geprägten Sportvereinen sowie in Vereinen, die Geselligkeit explizit fördern und nicht primär auf sportlichen Erfolg ausgerichtet sind, Vereinsaustritte unwahrscheinlicher. Vereine mit diesen Strukturgegebenheiten erhöhen die Stabilität der Mitgliedschaftsbeziehungen und zwar unabhängig von den individuellen Eigenschaften der Mitglieder. Damit bestätigt sich zunächst die Annahme, dass Mitglieder städtischer Sportvereine vielfältigere und attraktivere $\mathrm{Al}$ ternativen der Freizeitgestaltung zur Verfügung haben, so dass unter diesen Bedingungen für die Vereinsmitglieder ein Austritt weniger kostspielig (im Sinne adäquater Alternativen) sein dürfte. Weiterhin zeigt sich, dass der Sportverein als Ort der Geselligkeit nicht nur Werte des sozialen Miteinanders und solidarischen Verhaltens vermittelt, sondern auch als Katalysator der Stabilität der Mitgliedschaft wirkt, sofern entsprechende Gelegenheiten zur Verfügung stehen. Geselligkeit im Verein vermag demnach Anreize zur (dauerhaften) vereinsmäßigen Bündelung individueller Interessen zu schaffen, die in anderen sozialen Kontexten offensichtlich so nicht (mehr) zu finden sind. Im Zusammenhang mit der Mitgliederbindung scheint damit gerade jene vereinskulturelle Orientierung von Vereinen bedeutsam, die im Zuge von Individualisierungstendenzen oder Modernisierung von Vereinsangeboten gern als überholt erachtet wird. Der destabilisierende Einfluss des Vereinsziels „Erzielung sportlicher Erfolge“ ist in zweierlei Richtungen zu deuten: Einerseits könnte dies damit zusammenhängen, dass die Verfolgung (klassischer) Erfolgs- und Leistungsziele bei Mitgliedern, die bezüglich der Sportausübung primär an breitensportlichen Angeboten interessiert sind, verstärkt zu Ziel-Interessen-Divergenzen und damit zu einer Destabilisierung der Mitgliederbindung führen. Andererseits stellen für Wettkampfsportler sportliche Erfolge durchaus einen wichtigen Bindungsfaktor dar (Nagel \& Vogel, 2012). Aufgrund der hohen Relevanz dieser Ziele - dies zeigen die offenen Antworten unserer Studie bezüglich den Ursachen zum Vereinsaustritt - streben Mitglieder bei ausbleibendem Erfolg vielfach einen Wechsel in Vereine mit höherer sportlicher Performance und besseren sportlichen Perspektiven an. Auch wenn der Faktor Mitgliedsbeitrag im Gesamtmodell nicht signifikant ist, so scheint dieser in Bezug auf die Mitgliederbindung durchaus erklärungsrelevant zu sein. Höhere Mitgliedsbeiträge dürften das Austrittsrisiko insbesondere dann erhöhen, wenn auf Seiten der Mitglieder elastische Nachfragestrukturen (z. B. durch günstigere Konkurrenzangebote, begrenzte Haushaltsbudgets) bestehen. Die Ergebnisse der vorliegenden Studie zeigen überdies, dass die Vereinsgröße ohne systematische Wirkung auf die individuelle Bindungsentscheidung eines Vereinsmitglieds bleibt. Dies deutet darauf hin, dass nicht die organisatorische Einheit des formalen Vereins als Bezugspunkt für das Mitgliedschaftsverhältnis gilt, sondern die sozial überschaubare Einheit der Sportgruppe oder der Abteilung, die dann quasi als Vereine im Verein fungieren (Nagel et al. 2004). Weiterhin bleiben die Strukturmerkmale Vereinsalter, Angebotsvielfalt und der Grad der Professionalisierung sowie die Vereinsziele wie Tradition, 
Offenheit für neue Entwicklungen und Kooperationsbereitschaft ohne Einfluss. Dies ist insofern überraschend, da einerseits die Pflege der Vereinstradition als stabilisierender und andererseits die Offenheit eher als destabilisierender Faktor der Mitgliedschaftsverhältnisse akzentuiert werden. Insgesamt wird deutlich, dass die Mehrebenenperspektive einen wichtigen Baustein mit Blick auf die Konzeptualisierung einer allgemeinen „Theorie des Vereins" darstellt, wobei die kontextspezifischen Zwecksetzungen (hier: Sportangebot für die Mitglieder) durchaus strukturelle Unterschiede vermuten lassen, die sich wiederum spezifisch auf das individuelle Handeln von Vereinsmitgliedern auswirken können.

\section{Grenzen der Studie und Forschungsausblick}

Die vorliegende empirische Analyse stellt einen ersten Schritt zur Verknüpfung von Individualdaten und korrespondierenden Strukturdaten innerhalb der Sportvereinsforschung dar. Hierbei sind die Grenzen der Analyse zu berücksichtigen, die zugleich Forschungsperspektiven für weiterführende Studien eröffnen: Unsere Analyse beschränkt sich auf das Austrittsrisiko als das Maß für die Mitgliederbindung. Daher sollten in künftigen Studien auch ehemalige Vereinsmitglieder (Drop-outs) untersucht werden, die ihre Mitgliedschaft vor Kurzem (z. B. in den letzten 12 Monaten) beendet haben. Weiterhin sind in künftigen Studien zielführend und systematisch Erweiterungen vorzunehmen und bislang nicht untersuchte individuelle Parameter (z. B. Nutzen möglicher Alternativen, Kosten des Vereinsaustritts) bei der Analyse der Mitgliederbindung zu berücksichtigen. In Bezug auf die herangezogenen Strukturmerkmale ist anzumerken, dass die strukturellen Unterschiede zwischen den Vereinen aufgrund der zugrunde liegenden Fallzahl womöglich nicht hinreichend groß sind (zu homogene Vereinsauswahl). In weiterführenden Studien sollten daher größere Stichprobenumfänge, v. a. auf der Kontextebene (Zahl der Vereine) generiert werden. Zudem basieren die zugrunde liegenden Strukturdaten der Vereine auf den Selbstauskünften von Vereinsfunktionären, so dass z. B. Abweichungen zwischen anvisierter inhaltlicher Ausrichtung einerseits und Wahrnehmung durch die Mitglieder andererseits nicht auszuschließen sind. Abschließend stellt sich die Frage nach der Generalisierbarkeit der Befunde über Schweizer Sportvereine hinaus. Vor dem Hintergrund der bereits angesprochenen strukturellen Unterschiede von Sportvereinen in der Schweiz und in Deutschland, scheint eine Übertragbarkeit der Befunde auf deutsche Sportvereine nur bedingt möglich. Deshalb wäre als ein weiterer wichtiger Anknüpfungspunkt im Sinne der Generalisierbarkeit, eine Überprüfung der Robustheit der postulierten $\mathrm{Zu}$ sammenhänge in anderen Sportsystemen mit unterschiedlichen Strukturbedingungen des Vereinssports angezeigt.

\section{Korrespondenzadresse}

\section{Dr. T. Schlesinger}

Institut für Sportwissenschaft

Universität Bern

Bremgartenstrasse 145, 2012 Bern

torsten.schlesinger@ispw.unibe.ch

Interessenkonflikt. Der korrespondierende Autor gibt für sich und seinen Koautor an, dass kein Interessenkonflikt besteht.

\section{Literatur}

Baur, J., \& Braun, S. (2001). Der vereinsorganisierte Sport in Ostdeutschland. Köln: Sportverlag Strauß.

Baur, J., \& Burrmann, U. (2003). Der jugendliche Sporthopper als "moderne" Sozial figur? In J. Baur \& S. Braun (Hrsg.), Integrationsleistungen von Sportvereinen als Frei willigenorganisationen (S. 549583). Aachen: Meyer \& Meyer.

Bette, K.-H. (1993). Sport und Individualisierung. Spectrum der Sportwissenschaften, 5, 34-55.

Braun, S. (2001). Putnam und Bourdieu und das soziale Kapital in Deutschland. Der rhetorische Kurswert einer sozialwissenschaftlichen Kategorie. Leviathan, 29, 337-354.

Braun, S. (2003). Leistungserstellung in freiwilligen Vereinigungen. Über "Gemeinschaftsarbeit" und die „Krise des Ehrenamts". In J. Baur \& S. Braun (Hrsg.), Integrationsleistungen von Sportvereinen als Freiwilligenorganisationen (S. 191-241). Aachen: Meyer \& Meyer.

Braun, S., \& Nagel, M. (2005). Zwischen Solidargemeinschaft und Dienstleistungsorganisation. Mitgliedschaft, Engagement und Partizipation im Sportverein. In T. Alkemeyer, B. Rigauer \& G. Sobiech (Hrsg.), Organisationsentwicklungen und De-Institutionalisierungsprozesse im Sport (S. 123-150). Schorndorf: Hofmann.

Breuer, C. (Hrsg.). (2009). Sportentwicklungsbericht 2007/2008. Analyse zur Situation der Sportvereine in Deutschland. Köln: Sportverlag Strauß.
Breuer, C. (Hrsg.). (2011). Sportentwicklungsbericht 2009/2010. Analyse zur Situation der Sportvereine in Deutschland. Köln: Sportverlag Strauß.

Bühlmann, M., \& Freitag, M. (2004). Individuelle und Kontextuelle Determinanten der Teilhabe an Sozialkapital. Kölner Zeitschrift für Soziologie und Sozialpsychologie, 56, 326-349.

Büschges, G., \& Abraham, M. (1997). Einführung in die Organisationssoziologie. Stuttgart: Teubner.

Cachay, K. (1988). Perspektiven der künftigen Entwicklung von Sportvereinen und Sportverbänden. In $\mathrm{H}$. Digel (Hrsg.), Sport im Verein und Verband: Historische, politische und soziologische Aspekte (S. 219-233). Schorndorf: Hofmann.

Cachay, K., Thiel, A., \& Meier, H. (2001). Der organisierte Sport als Arbeitsmarkt. Eine Studie zu Erwerbspotenzialen in Sportverein und Sportverbänden. Schorndorf: Hofmann.

Chen, L. (2004). Membership incentives: Factors affecting individuals' decisions about participation in athletics-related professional associations. Journal of Sport Management, 18, 111-131.

Coleman, J. S. (1974). Power and the structure of society. New York: Norton.

Coleman, J. S. (1990). Foundations of social theory. Cambridge, MA: Belknap.

Dürr, F. (2009). Faktoren der Mitgliederzufriedenheit im Sportverein. Bedeutsamkeit von Vereinsbewertungsmerkmalen für die Zufriedenheit sportlich aktiver Mitglieder ohne ehrenamtliches und berufliches Engagement. Saarbrücken: SVH.

Emrich, E. (2009). Organisationstheoretische Besonderheiten des Sports. In C. Breuer \& A. Thiel (Hrsg.), Handbuch Sportmanagement (2. Aufl., S. 103121). Schorndorf: Hofmann.

Emrich, E., Papathanassiou, V., \& Pitsch, W. (1999). Sportvereine im Blickpunkt. Strukturelemente, Umweltverflechtungen und Selbstverständnis saarländischer Sportvereine. St. Ingbert: Röhrig.

Emrich, E., Pitsch, W., \& Papathanassiou, V. (2001). Die Sportvereine. Ein Versuch auf empirischer Grundlage. Schorndorf: Hofmann.

Esser, H. (1999). Soziologie. Spezielle Grundlagen. Bd. 1: Situationslogik und Handeln. Frankfurt am Main: Campus.

Gieß-Stüber, P., \& Rücker, V. (2003). Drop-Out analysieren, verstehen und vermeiden - Die Zukunft liegt in der Mitgliedergewinnung und Mitgliederbindung. In A. Woll (Hrsg.), Miteinander lernen, forschen, spielen: Zukunftsperspektiven für Tennis (S. 7283). Hamburg: Czwalina.

Heinemann, K. (1995). Einführung in die Ökonomie des Sports. Schorndorf: Hofmann.

Heinemann, K. (2004). Sportorganisationen. Verstehen und Gestalten. Schorndorf: Hofmann.

Hirschman, A. O. (1974). Abwanderung und Widerspruch (L. Walentik, Übers.). Tübingen: Mohr. (Originalarbeit erschienen 1970).

Horch, H.-D. (1992). Geld, Macht und Engagement in freiwilligen Vereinigungen. Grundlage einer Wirtschaftssoziologie von Non-Profit-Organisationen. Berlin: Duncker \& Humblot.

Hox, J. J. (2002). Multilevel analysis: Techniques and applications. Mahwah: Lawrence Erlbaum.

Klenk, C. (2011). Ziel-Interessen-Divergenzen in freiwilligen Sportorganisationen. Eine akteurtheoretische Analyse der Ursachen und Auswirkungen. Hamburg: Czwalina.

Lamprecht, M., Fischer, A., \& Stamm, H.-P. (2012). Die Schweizer Sportvereine - Strukturen, Leistungen, Herausforderungen. Zürich: Seismo.

Lenk, H. (1972). Materialien zur Soziologie des Sportvereins. Ahrensburg: Czwalina. 
Meyer, J. P., Stanley, D. J., Herscovitch, L., \& Topolnytsky, L. (2002). Affective, continuance, and normative commitment to the organizations: A meta-analysis of antecedents, correlates, and consequences. Journal of Vocational Behavior, 61, 20-52.

Nagel, S. (2006a). Sportvereine im Wandel. Akteurtheoretische Analysen zur Entwicklung von Sportvereinen. Schorndorf: Hofmann.

Nagel, S. (2006b). Mitgliederbindung in Sportvereinen - Ein akteurtheorteisches Modell. Sport und Gesellschaft, 3, 33-56.

Nagel, S. (2007). Akteurtheoretische Analyse der Sportvereinsentwicklung - ein theoretisch-methodischer Bezugsrahmen. Sportwissenschaft, 37, 186201.

Nagel, S., Conzelmann, A., \& Gabler, H. (2004). Sportvereine Auslaufmodell oder Hoffnungsträger. Tübingen: Attempto.

Nagel, S., \& Vogel, M. (2012). Sozioökonomische Analyse der Mitgliederbindung von Jugendlichen in Fußballvereinen In G. Trosien (Hrsg.), Ökonomie der Sportspiele (S. 173-192). Schorndorf: Hofmann.

Nagel, S., \& Schlesinger, T. (2012). Sportvereinsent wicklung - Ein Leitfaden zur Planung und Durchführung von Veränderungsprozessen. Bern u. a.: Haupt Verlag.

Raudenbush, S. W., \& Bryk, A. S. (2002). Hierarchical linear models: Applications and data analysis methods. Thousand Oaks: Sage.

Rittner, V. (1986). Sportvereine und gewandelte Bedürfnisse. In G. A. Pilz (Hrsg.), Sport und Verein (S. 4355). Reinbek: Rowohlt

Sandler, T., \& Tschirhart, J. T. (1980). The economic theory of clubs: An evaluative survey. Journal of Economic Literature, 18, 1481-1521.

Schimank, U. (2005). Der Vereinssport in der Organisationsgesellschaft: Organisationssoziologische Perspektiven auf ein Spannungsverhältnis. In T. Alkemeyer, B. Rigauer \& G. Sobiech (Hrsg.), Organisationsentwicklungen und Delnstitutionalisierungsprozesse im Sport (S. 21-44). Schorndorf: Hofmann.
Schlagenauf, K. (1977). Sportvereine in der Bundesrepublik Deutschland. Teil I: Struktur elemente und Verhaltensdeterminanten im organisierten Freizeitbereich. Schorndorf: Hofmann.

Schlesinger, T., \& Nagel, S. (2011). „Freiwilliges Engagement im Sportverein ist Ehrensache! " - Ein Modell zur Analyse der Mitarbeitsentscheidungen in Sportvereinen. Sport und Gesellschaft, 8, 3-27.

Snijders, T., \& Bosker, R. (1999). Multilevel analysis: An introduction to basic and advanced multilevel modeling. London: Sage.

Stigler, G. J., \& Becker, G. S. (1977). De gustibus non est diputandum. American Economic Review, 67, 7690.

Strob, B. (1999). Dervereins- und verbandsorganisierte Sport:Ein Zusammenschluss von (Wahl-)Gemeinschaften? Münster: Waxmann.

Timm, W. (1979). Sportvereine in der Bundesrepublik Deutschland. Teil II: Organisations-, Angebotsund Finanzstruktur. Schorndorf: Hofmann.

Vanberg, V. (1978). Kollektive Güter und Kollektives Handeln - Die Bedeutung neuerer ökonomischer Theorieentwicklungen für die Soziologie. Kölner Zeitschrift für Soziologie und Sozialpsychologie, 30, 652-679.

Wicker, P. (2009). Perspektiven und Grenzen der Beitragsfinanzierung von Sportvereinen - Eine Analyse zur Theorie und Empirie der Beitragselastizität im Sportverein. Dissertation. Köln: Deutsche Sporthochschule Köln.

Wicker, P., \& Breuer, C. (2011). Scarcity of resources in German non-profit sport clubs. Sport Management Review, 14, 188-201.

Woll, A. (2003). Bindung an den Verein - Kundenzufriedenheit im Tennis. In A. Woll (Hrsg.), Miteinander lernen, forschen, spielen: Zukunftsperspektiven für Tennis (S. 121-136). Hamburg: Czwalina. 